\title{
Charge transport effects in field emission from carbon nanotube-polymer composites
}

\author{
R. C. Smith and J. D. Carey ${ }^{\text {a) }}$ \\ Advanced Technology Institute, University of Surrey, Guildford GU2 7XH, United Kingdom \\ R. J. Murphy, W. J. Blau, and J. N. Coleman \\ School of Physics, Trinity College Dublin, Dublin 2, Ireland \\ S. R. P. Silva \\ Advanced Technology Institute, University of Surrey, Guildford GU2 7XH, United Kingdom
}

(Received 31 August 2005; accepted 21 November 2005; published online 21 December 2005)

\begin{abstract}
Electron field emission measurements have been made on multiwall arc discharge carbon nanotubes embedded in a conjugated polymer host. Electron emission at low nanotube content is observed and attributed to an enhancement of the applied electric field at the polymer/nanotube/vacuum interface where the electron supply through the film is attributed to fluctuation induced tunneling in a disordered percolation network. A high network resistance is attributed to a polymer coating surrounding each nanotube, resulting in high resistance nanotube-polymer-nanotube tunnel junctions. The potential use of carbon nanotube-polymer composites for field emission based displays is also discussed. (C) 2005 American Institute of Physics. [DOI: 10.1063/1.2158023]
\end{abstract}

Electron field emission based displays (FEDs) are highly attractive from a technological viewpoint for the next generation of displays. ${ }^{1}$ Considerable research is currently being undertaken to find alternative cathode materials that emit electrons at relatively low applied electric fields. The discovery $^{2}$ of carbon nanotubes (CNTs) inevitably led to the use of CNTs as potential electron sources ${ }^{3}$ and the subsequent development of a prototype FED. ${ }^{4}$ In addition, electron emission from carbon nanofibers grown on low temperature substrates has also been demonstrated. ${ }^{5}$ Optimizing emission from CNTs is an important technological goal due to the relatively high current production costs of nanotubes. To that end, the previous report of emission from CNTs, with mass fractions of $11 \%-33 \%$ embedded in a polystyrene matrix, is therefore important since the use of a composite structure potentially allows a lower concentration of nanotubes to be used. ${ }^{6}$

The concept of an emitter based material embedded in a host matrix can be originally traced back to the pioneering work of Latham. ${ }^{7}$ This approach has been recently revived by the incorporation of conductive graphitic particles into a nonconductive epoxy matrix to form metal-insulator-metalinsulator-vacuum cathodes. ${ }^{8}$ In these electrically inhomogeneous cathodes, tailoring the concentration of the conductive phase, as a means to control charge transport, is an important issue. For flat metals, while there is an abundance of electrons, the absence of a surface geometric enhancement factor coupled with a high surface potential barrier (work function) results in the need for a high electric field $(>200 \mathrm{~V} / \mu \mathrm{m})$ to be applied for emission. In DLC films, emission has been described in terms of transport of electrons by hopping between conductive $s p^{2}$ clusters. ${ }^{9}$ From these examples, it is evident that charge transport and emission of electrons are intrinsically linked. In this letter we report on the field emission characteristics of multiwall carbon nanotube-conjugated polymer composites as a function of nanotube loading. We

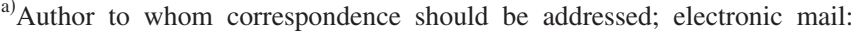
david.carey@surrey.ac.uk show how the threshold field for emission decreases with nanotube loading and the transport of charge through a disordered percolation network.

Composite solutions of conjugated polymer $\operatorname{poly}(m-$ phenylenevinylene-co-2,5-dioctyloxy- $p$-phenylenevinylene) (PmPV) and Kraetschmer arc discharge multiwall nanotubes (MWNTs) were produced using toluene as a solvent at Trinity College Dublin and described in detail elsewhere. ${ }^{10}$ The MWNTs possessed an average diameter and length of $20 \mathrm{~nm}$ and $1 \mu \mathrm{m}$, respectively. Composite solutions were sonicated with a high power sonic tip for $1 \mathrm{~min}$ and then, subsequently, in a low power sonic bath for $1 \mathrm{~h}$. The solutions were then allowed to stand undisturbed for $24 \mathrm{~h}$ to remove any nonnanotube impurities. Decantation then yielded composite solutions free from graphitic impurities. Films of $80 \mathrm{~nm}$ thickness were spin coated using a Convac 1001 spin coater onto gold coated glass substrates. The FE characteristics of these films were examined in an evacuated two terminal arrangement using a phosphor coated anode. ${ }^{11}$ A polytetrafluoroethylene spacer of $100 \mu \mathrm{m}$ thickness was used and the macroscopic threshold field, $E_{\mathrm{th}}$, is defined as the applied electric field which gives an emission current of $10 \mathrm{nA}$.

The FE current-applied electric field characteristics for a series of samples ranging from $7 \%$ nanotube loading (sample A) to $0.44 \%$ loading (sample E) are shown in Fig. 1(a). Emission from a PmPV control sample (no nanotubes) did not occur until an applied field of over $40 \mathrm{~V} / \mu \mathrm{m}$ and was attributed to breakdown of the film. The Fowler-Nordheim (F-N) representation of the current-field characteristics of Fig. 1(a) are presented in Fig. 1(b). It should be noted that we have also analyzed (not presented here) the FE characteristics using both a space charge limited current model and a Schottky model and found the best results (as judged by the quality of the fit) to be via the Fowler-Nordheim model. The variation of the field enhancement factor, $\beta$, obtained from the F-N analysis assuming a work function of $5 \mathrm{eV}$, as a function of nanotube mass fraction, along with the variation of the measured threshold fields, $E_{\mathrm{th}}$, are also shown in Fig. 2(a). It is apparent that the enhancement factor does not vary significantly with mass fraction, increasing only moderately 

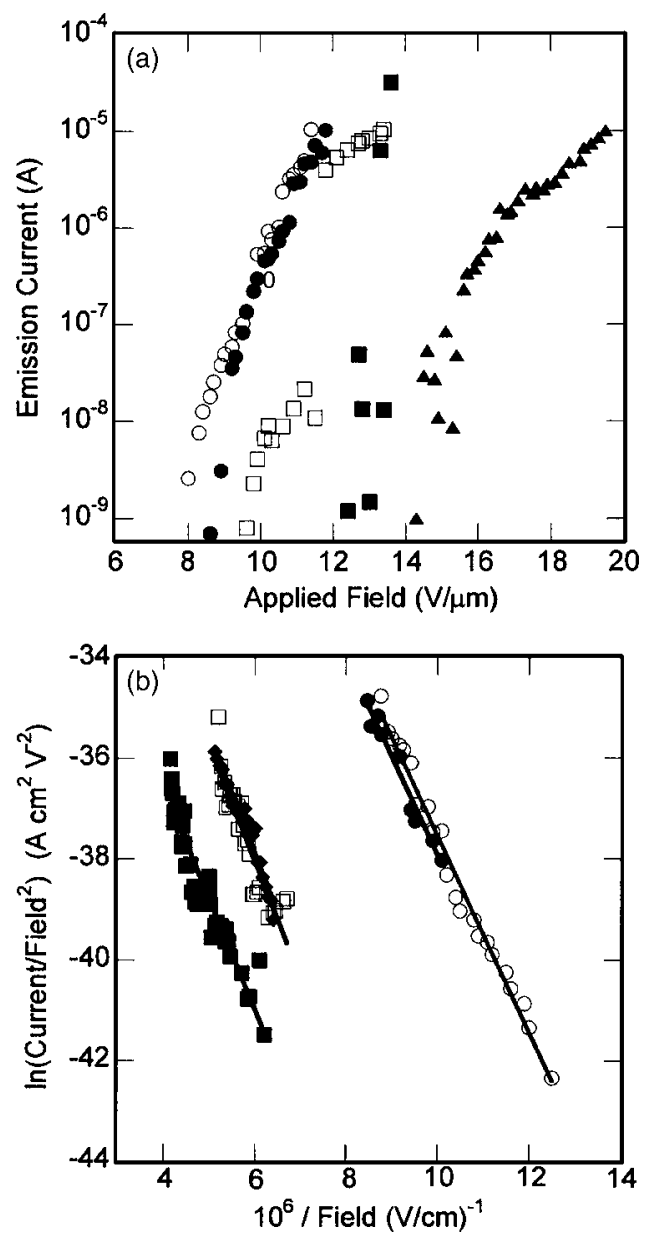

FIG. 1. (a) Field emission current-field characteristics and (b) FowlerNordheim representation for CNT-polymer composites with different loadings: $7 \%(\bigcirc), 3.5 \%(\bullet), 1.75 \%(\square), 0.88 \%(\square)$, and $0.44 \%(\mathbf{\Delta})$. Not all data points are shown for clarity.

from 330 (0.44\% loading) up to 390 (7\% loading). Over the same range of nanotube loading $E_{\mathrm{th}}$, decreases from $14.5 \mathrm{~V} / \mu \mathrm{m}$ ( $0.44 \%$ loading) to $8.4 \mathrm{~V} / \mu \mathrm{m}$ (7\% loading). The solid line in Fig. 2(a), represents a power law dependence with loading, with an exponent of -0.21 . The observed $d e$ crease of $E_{\mathrm{th}}$ with increasing loading, differs from the results of the study by Poa et al. who reported an increase of $E_{\text {th }}$ with increasing weight fractions of both undoped and B doped multiwall nanotubes embedded in polystyrene (PS), ${ }^{6}$ however, with considerably larger CNT mass fractions (11-33 wt \%).

Armed with a knowledge of the macroscopic threshold field and the field enhancement factor, $\beta$, it is possible to calculate the threshold local electric field, $F_{\text {th }}$, given by $\beta E_{\text {th }}$, for each sample. Figure 2(b) shows the variation of $F_{\text {th }}$ for CNTs embedded in PmPV and for both series of Ref. 6 of doped and undoped nanotubes embedded in PS. It is evident that the local threshold fields for the samples embedded in PmPV do not vary strongly with mass fraction, with a typical local field of about $3.3-4.8 \mathrm{~V} / \mathrm{nm}$. The solid line is power law fit with mass fraction with an exponent of -0.13 . By contrast, the local fields for the samples embedded in PS did vary significantly with CNT loading, decreasing from 61(79) $\mathrm{V} / \mathrm{nm}$ for the undoped (B doped) samples at a loading of $11 \mathrm{wt} \%$ down to $14(13) \mathrm{V} / \mathrm{nm}$ at $33 \mathrm{wt} \%$. The lack of any significant variation in the local fields for the CNTs in PmPV indicates that similar emission conditions are present Downloaded 19 Mar 2009 to 134.226.1.229. Redistribution subjec
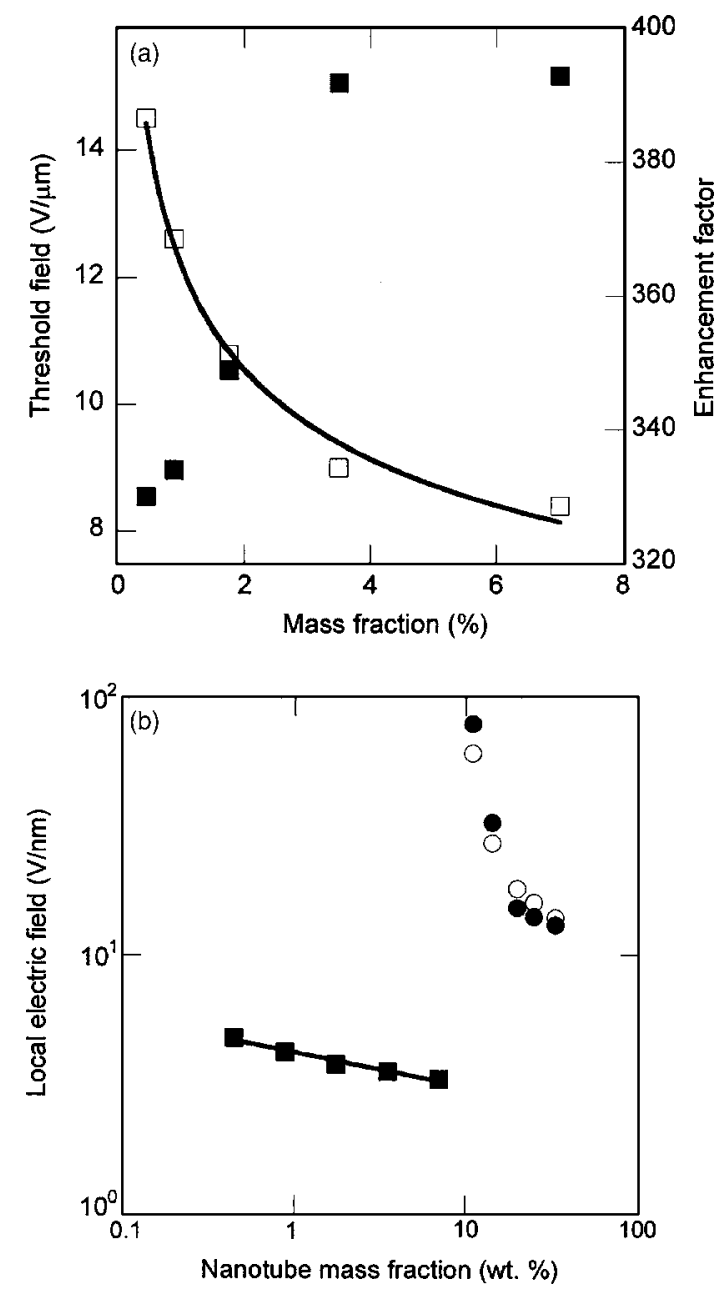

FIG. 2. (a) Variation of threshold field ( $\square$ ) and enhancement factor ( $\square$ ) for CNT-PmPV composites. (b) Variation of the calculated local electric field for CNT-PmPV (ם), undoped MWNT (O), and B doped MWNTs $(\bullet)$ in PS.

in each sample at the PmPV/CNT/vacuum triple junction. This suggests that the nanotubes in these films experience a similar electrostatic environment, which changes little with loading. The local threshold field should be independent of nanotube loading, so the small increase in $F_{\text {th }}$ as the nanotube loading decreases reflects the overall resistive nature of the network. The observation of a power law dependence of both $E_{\mathrm{th}}$ and $F_{\mathrm{th}}$, with loading, can be attributed to percolation transport through the films and the need to tunnel through the potential barrier at the front surface. By contrast, for both sets of films in PS, the strong variation of local field, which manifests itself as an apparent linear increase in applied threshold fields, suggests that the electrostatic environment is significantly different as the polymer loading increases. It is known that screening effects due to the proximity of neighbouring nanotubes results in high threshold fields required for emission. ${ }^{12}$

An immediate question arises as to what is the controlling or limiting factor for emission from the CNTs embedded in PmPV. As discussed previously, emission can be controlled by the transport of charge through the film, as well as the properties of the emitters at the front surface. The transport properties of nanotube-PmPV composites, with mass fraction, $p$, have been reported elsewhere ${ }^{10}$ where the conductivity, $\sigma$, of the films follows a scaling law of the form
to AlP license or copyright; see http://apl.aip.org/apl/copyright.jsp 


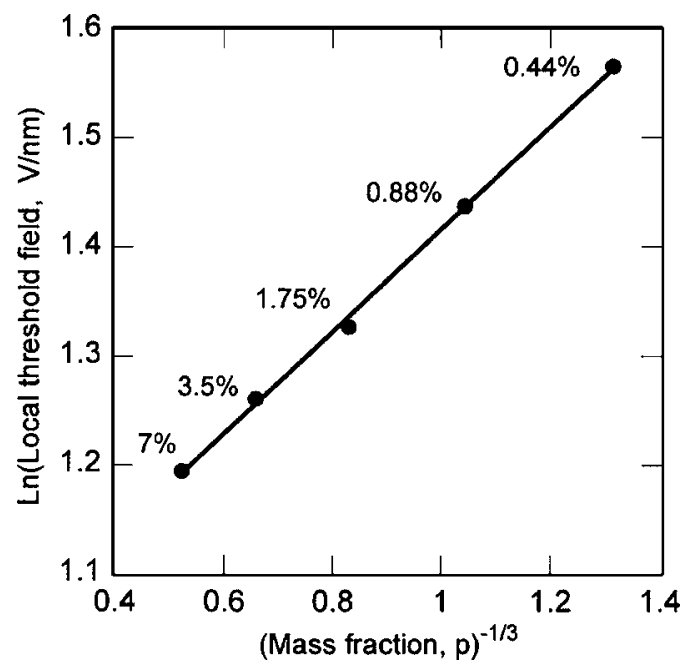

FIG. 3. Variation of $\ln$ (local threshold field) with $p^{-1 / 3}$ for CNT-PmPV composites demonstrating fluctuation induced tunneling. The corresponding nanotube loadings are also indicated.

$$
\sigma \sim \sigma_{0}\left(p-p_{\mathrm{c}}\right)^{t}
$$

where the critical loading $p_{\mathrm{c}}$ was found to be $0.055 \%$ and the value of the critical exponent $t=1.36$. Such low values of critical loading, may at first sight appear unusual since it is known that the critical volume fractions (CVF) for spheres is around $16 \%$. However, a recent Monte Carlo ${ }^{13}$ simulation study by Foygel et al. has showed that if nanotubes are considered to be capped three dimensional nonaligned interpenetrating conductive cylinders with a high aspect ratio, $a$, then an upper limit to the value of CVF is approximately given by $\sim 0.6 / a$. The high aspect ratio of the fillers can significantly reduce the value of CVF. It is instructive to estimate the effective resistance of the percolation network, $R_{0}$, using the approach of Foygel et al., where it was shown that the conductivity prefactor $\sigma_{0}$ can be expressed as

$$
\sigma_{0}=\left(R_{0} l p_{\mathrm{c}}^{t}\right)^{-1}
$$

where $l$ is the average length of the nanotubes. Using the known values ${ }^{10}$ of conductivity, threshold loading $p_{\mathrm{c}}$ and exponent $t$, it can be shown that the resistance of the percolation network is about $5.7 \times 10^{13} \Omega$. The value of network resistance is considerably higher than the intrinsic resistance of the nanotubes themselves but is comparable to the value of network resistance $\left(10^{14} \Omega\right)$ found for $1 \%$ vol. SWNTs embedded in a polyimide matrix. ${ }^{13}$ Such high values of resistance are attributed to a polymer coating that surrounds each of the nanotubes and acts as a high tunnel junction. Variations in the thickness of the polymer coatings, as well as nanotube-nanotube separation, varies the tunnel barrier and this fluctuation induced tunneling ${ }^{14}$ (FIT) determines the intertube transport. A characteristic of FIT is that the log(conductivity) within the film should vary as $-p^{-1 / 3}$. In general, whilst it is not possible to assign a field emission "conductivity," if the limiting step for emission is the transport through the film, with emission determined largely by a constant enhancement factor, then $F_{\text {th }}$ should reflect the transport through the film and exhibit a $p^{-1 / 3}$ dependence. Figure 3 clearly demonstrates such a linear variation of the natural logarithm of local threshold field with $p^{-1 / 3}$.
In this regard, it is possible to explain the electron emission mechanism for CNT-PmPV composites as follows: emission of the electrons from the surface at the nanotube/ polymer/vacuum triple junction occurs due to an enhancement of the applied field brought about by the aspect ratio of the nanotube, each of which will have a thin polymer coating. This enhancement factor does not have a strong variation with loading. As the mass fraction of nanotubes increases, a greater density of nanotube-nanotube contacts is made, increasing the overall charge transport of carriers via FIT through the tunnel barriers, which result from the polymer coating around each nanotube. Replenishment of the electrons occurring by a percolation controlled charge transport through the disordered nanotube network.

Finally, in the study of Alexandrou et al. turn-on fields of $3 \mathrm{~V} / \mu \mathrm{m}$ were reported ${ }^{15}$ but from a sample consisting of up to 33 wt \% of single wall nanotubes embedded in poly(3octylthiophene). Since single wall nanotubes are expensive to produce, the use of MWNTs at low mass fractions offers the opportunity to reduce cost. Furthermore, spin casting allows much large area cathodes to be produced.

In summary, we have studied the field emission characteristics of spin cast MWNTs embedded in PmPV polymer with low mass fractions. It has been shown that field emission from these samples is determined by the charge transport, via fluctuation induced tunneling, through a disordered network in the film. The ability to use lower mass fractions of MWNTs (less than 10\%) is important for the development of nanotube based FEDs.

The authors would like to acknowledge funding from the EPSRC (UK) via the award of an Advanced Research Fellowship to J.D.C. and via a Portfolio Partnership award.

\footnotetext{
${ }^{1}$ See, for example, J. E. Jaskie, MRS Bull. 21, 59 (1996).

${ }^{2}$ S. Iijima, Nature (London) 354, 56 (1991).

${ }^{3}$ J.-M. Bonard, J.-P. Salvetat, T. Stockli, W. A. De Heer, L. Forro, and A. Chatelin, Appl. Phys. Lett. 73, 918 (1998).

${ }^{4}$ W. B. Choi, D. S. Chung, J. H. Kang, H. Y. Kim, Y. W. Jin, I. T. Han, Y. H. Lee, J. E. Jung, N. S. Lee, G. S. Park, and J. M. Kim, Appl. Phys. Lett. 75, 3129 (1999).

${ }^{5}$ R. C. Smith, J. D. Carey, C. H. P. Poa, D. C. Cox, and S. R. P. Silva, J. Appl. Phys. 95, 3153 (2004).

${ }^{6}$ C. H. Poa, S. R. P. Silva, P. C. P. Watts, W. K. Hsu, H. W. Kroto, and D. R. M. Walton, Appl. Phys. Lett. 80, 3189 (2002); C. H. P. Poa, R. C. Smith, S. R. P. Silva, P. C. P. Watts, W. K. Hsu, H. W. Kroto, and D. R. M. Walton, J. Vac. Sci. Technol. B 21, 1715 (2003).

${ }^{7}$ R. V. Latham, High Voltage Vacuum Insulation, The Physical Basic (Academic, London, 1981).

${ }^{8}$ A. P. Burden, H. E. Bishop, M. Brierley, J. M. Friday, C. Hood, P. G. A. Jones, A. Y. Khazov, W. Lee, R. J. Riggs, V. L. Shaw, and R. A. Tuck, J. Vac. Sci. Technol. B 18, 900 (2000).

${ }^{9}$ J. D. Carey, R. D. Forrest, and S. R. P. Silva, Appl. Phys. Lett. 78, 2339 (2001).

${ }^{10}$ B. E. Kilbride, J. N. Coleman, J. Fraysse, P. Fournet, M. Cadek, A. Drury, S. Hutzler, S. Roth, and W. J. Blau, J. Appl. Phys. 92, 4024 (2002).

${ }^{11}$ R. D. Forrest, A. P. Burden, S. R. P. Silva, and X. Shi, Appl. Phys. Lett. 73, 3784 (1998).

${ }^{12}$ L. Nilsson, O. Groening, C. Emmenegger, O. Kuettel, E. Schaller, L. Schlapbach, H. Kind, J.-M. Bonard, and K. Kern, Appl. Phys. Lett. 76, 2071 (2000).

${ }^{13}$ M. Foygel, R. D. Morris, D. Anez, S. French, and V. L. Sobolev, Phys. Rev. B 71, 104201 (2005).

${ }^{14}$ P. Sheng, E. K. Sichel, and J. I. Gittleman, Phys. Rev. Lett. 40, 1197 (1978).

${ }^{15}$ I. Alexandrou, E. Kymakis, and G. A. J. Alexandrou, Appl. Phys. Lett. 80, 1435 (2002).
} 